\title{
Biossorção do corante vermelho escarlate direto por bagaço de mandioca
}

\author{
Biosorption of direct scarlet red dye by cassava bagasse \\ Biosorción de colorante rojo escarlata directo por bagazo de yuca
}

Recebido: 16/03/2021 | Revisado: 26/03/2021 | Aceito: 26/03/2021 | Publicado: 04/04/2021

\author{
Ingridy Alessandretti \\ ORCID: https://orcid.org/0000-0002-2322-3667 \\ Universidade de Passo Fundo, Brasil \\ E-mail: ingridyalessandretti7@gmail.com \\ Regiane Ribeiro de Jesus \\ ORCID: https://orcid.org/0000-0003-2842-4692 \\ Universidade do Estado de Mato Grosso, Brasil \\ E-mail: mailto:regiannezoomp@hotmail.com \\ Sumaya Ferreira Guedes \\ ORCID: https://orcid.org/0000-0002-1676-6030 \\ Universidade do Estado de Mato Grosso, Brasil \\ E-mail: su_sumaya@yahoo.com.br \\ Raquel Aparecida Loss \\ ORCID: https://orcid.org/0000-0002-6022-7552 \\ Universidade do Estado de Mato Grosso, Brasil \\ E-mail: raquelloss@unemat.br \\ Juliana Maria de Paula \\ ORCID: https://orcid.org/0000-0002-4960-1558 \\ Universidade do Estado de Mato Grosso, Brasil \\ E-mail: depaulajm@outlook.com \\ Claudineia Aparecida Queli Geraldi \\ ORCID: https://orcid.org/0000-0001-5255-9752 \\ Universidade do Estado de Mato Grosso, Brasil \\ E-mail: claudigeraldi@onda.com.br
}

\begin{abstract}
Resumo
A indústria têxtil faz uso de corantes nos processos de tingimento, gerando efluentes com potencial tóxico ao meio ambiente e seres humanos, se não tratados adequadamente. A biossorção é uma alternativa para remoção de corantes de matrizes aquosas, sendo uma técnica de baixo custo e efetiva, possibilitando ainda, o uso de resíduos agroindustriais. Portanto, esse trabalho objetivou avaliar a capacidade de remoção do corante vermelho escarlate direto utilizando como biossorvente o bagaço de mandioca, um resíduo amplamente gerado no Brasil. O biossorvente foi caracterizado quanto a sua área específica superficial. Inicialmente, testes preliminares foram realizados para obter as melhores condições de $\mathrm{pH}$, temperatura e velocidade de rotação, e posteriormente, foram realizados testes cinéticos e de equilibrio de adsorção. A modelagem matemática foi empregada a fim de compreender os mecanismos envolvidos na adsorção do corante. O bagaço de mandioca apresentou área superficial específica de $3,012 \mathrm{~m}^{2} \mathrm{~g}^{-1}$, com presença de microporos. Os ensaios de biossorção em batelada, obtiveram condições ótimas de operação em pH 2, $50{ }^{\circ} \mathrm{C}$ e $90 \mathrm{rpm}$. Na cinética, em $300 \mathrm{~min}$ obteve-se remoção de 84\%. Nas isotermas de adsorção, a capacidade máxima de adsorção em monocamada estimada pelo modelo de Langmuir foi de $25,1 \mathrm{mg} \mathrm{g}^{-1}$. Na modelagem matemática, ambos os modelos de Pseudo-primeira ordem, Pseudo-segunda ordem e Elovich, representam os dados cinéticos, sugerindo ocorrência de mais de um mecanismo no processo, enquanto nas isotermas, os modelos de Redlich-Peterson e Toth sugerem uma tendência ao modelo de Freundlich. Em geral, o bagaço de mandioca mostrou-se um adsorvente eficiente na remoção do corante em estudo.

Palavras-chave: Bagaço de mandioca; Biossorção; Corante têxtil.
\end{abstract}

\begin{abstract}
Textile industry uses dyes in the dyeing processes, generating effluents with potential toxicity to the environment and humans, if not treated adequately. Biosorption is an alternative for removing dyes from aqueous matrices, being a lowcost and effective technique, also allowing the use of agroindustrial wastes. Therefore, this work aimed to evaluate the capacity to remove the direct scarlet red dye using cassava bagasse as a biosorbent, a waste widely generated in Brazil. The biosorbent was characterized according to its specific surface area. Initially, preliminary tests were performed to obtain the best conditions of $\mathrm{pH}$, temperature, and speed of rotation. Kinetic and adsorption equilibrium tests were performed. Mathematical modeling was employed in order to understand the mechanisms involved in the adsorption of the dye. The cassava bagasse had a specific surface area of $3.012 \mathrm{~m}^{2} \mathrm{~g}^{-1}$, with the presence of micropores. The batch biosorption tests obtained optimal operating conditions at $\mathrm{pH} 2,50{ }^{\circ} \mathrm{C}$ and $90 \mathrm{rpm}$. In kinetics, removal of $84 \%$ was
\end{abstract}


achieved in $300 \mathrm{~min}$. In adsorption isotherms, the maximum monolayer adsorption capacity estimated by the Langmuir model was $25.1 \mathrm{mg} \mathrm{g}^{-1}$. In mathematical modeling, both Pseudo-first order, Pseudo-second order and Elovich models represent kinetic data, suggesting the occurrence of more than one mechanism in the process, whereas, in isotherms, the Redlich-Peterson and Toth models suggest a trend to the Freundlich model. In general, cassava bagasse proved to be an efficient adsorbent in removing the textile dye.

Keywords: Cassava bagasse; Biosorption; Textile dye.

\section{Resumen}

La industria textil hace uso de tintes en los procesos de tintura, generando efluentes con potencial tóxico para el medio ambiente y los seres humanos, si no se tratan adecuadamente. La biosorción es una alternativa para la remoción de colorantes de matrices acuosas, siendo una técnica de bajo costo y efectiva, permitiendo también el uso de residuos agroindustriales. Por lo tanto, este trabajo tuvo como objetivo evaluar la capacidad de eliminar el tinte rojo escarlata directo utilizando bagazo de yuca como biosorbente, un residuo ampliamente generado en Brazil. El biosorbente se caracterizó en términos de su superficie específica. Inicialmente se realizaron pruebas preliminares para obtener las mejores condiciones de $\mathrm{pH}$, temperatura y velocidad de rotación, y posteriormente se realizaron pruebas de equilibrio cinético y de adsorción. Se empleó un modelo matemático para comprender los mecanismos involucrados en la adsorción del tinte. El bagazo de yuca tuvo una superficie específica de $3.012 \mathrm{~m}^{2} \mathrm{~g}^{-1}$, con presencia de microporos. Las pruebas de biosorción por lotes obtuvieron condiciones óptimas de funcionamiento a $\mathrm{pH} 2,50{ }^{\circ} \mathrm{C} \mathrm{y} 90 \mathrm{rpm}$. En cinética, se logró una eliminación del 84\% en 300 min. En las isotermas de adsorción, la capacidad máxima de adsorción de monocapa estimada por el modelo de Langmuir fue de $25,1 \mathrm{mg} \mathrm{g}^{-1}$. En el modelado matemático, los modelos de Pseudoprimer orden, Pseudo-segundo orden y Elovich representan datos cinéticos, lo que sugiere la ocurrencia de más de un mecanismo en el proceso, mientras que en las isotermas, los modelos de Redlich-Peterson y Toth sugieren una tendencia al Freundlich modelo. En general, el bagazo de yuca demostró ser un adsorbente eficaz para eliminar el tinte en estudio. Palabras clave: Bagazo de yuca; Biosorción; Tinte textil.

\section{Introdução}

A indústria têxtil no Brasil é a segunda maior empregadora, de acordo com dados da Associação Brasileira da Indústria Têxtil e de Confecção (2015), no entanto, devido a esse destaque na produção de texteis, elevados volumes de efluentes são gerados, oriundos das etapas de tingimento, e o tratamento destes é um constante desafio (Marques et al., 2019). Os corantes presentes no processo têxtil, são compostos não biodegradáveis e estáveis, sendo que de 10-15\% não se fixam as fibras têxteis e são descartados nos efluentes (Gita, Hussan, \& Choudhury, 2017). Sendo assim, mesmo em concentrações baixas podem gerar risco a saúde humana e ao ecossistema, devido seu potencial carcinogênico, mutagênico e tóxico (Moussavi \& Mahmoudi, 2009).

Uma das técnicas utilizadas para remoção de contaminantes em meio aquoso, é a adsorção, que se destaca por ser um processo simples, de fácil operação e implementação, baixo custo e alta taxa de remoção (Rigueto et al., 2020a; Zhuo et al., 2017). A adsorção faz o uso de materiais adsorventes, os quais devem possuir característica como baixo custo e disponibilidade, propriedades texturais e físico-químicas adequadas, alta capacidade de adsorção e eficiência (Dotto \& McKay, 2020). Nesse contexto, resíduos provenientes de atividades agroindustriais, tem ganhado destaque como material adsorvente, devido a redução do custo do processo (Ahmad \& Danish, 2018; Rigueto et al, 2021a).

No Brasil em 2019, mais de 17 toneladas de mandioca foram produzidas, sendo que para cada 250-300 toneladas de raiz de mandioca processada, 280 toneladas de bagaço são gerados, com cerca de $85 \%$ de umidade. Portanto, devido à grande quantidade desses resíduos, a valorização destes se torna necessária (Escaramboni et al., 2018; FAO, 2019; Pandey et al., 2000).

O bagaço de mandioca é um material fibroso, o qual apresenta em torno de $50 \%$ de amido residual na matéria seca, logo por ser um resíduo vegetal, abundante, barato, renovável e biodegradável, torna-se um biossorvente alternativo para a remoção de contaminantes em meio aquoso, devido seus aspectos ambientais e econômicos que favorecem a adsorção (Alvarado et al., 2021; Polachini et al., 2016).

Nesse contexto, o presente trabalho objetivou avaliar a capacidade de remoção do corante vermelho escarlate direto utilizando como biossorvente o bagaço de mandioca. 


\section{Metodologia}

O biossorvente utilizado foi o bagaço de mandioca, coletado na região oeste do estado do Paraná, Brasil. O corante utilizado na pesquisa foi o vermelho escarlate direto (Texpal, Brasil) e todos os reagentes utilizados eram de grau analítico.

O resíduo foi submetido à secagem convectiva (CienLab, CE-480, Brasil) à $50^{\circ} \mathrm{C}$ por 24 h. Após, foi moído em moinho de martelo (SPLabor, SP-33, Brasil) com malha de \#2 a \#20 mesh.

\subsection{Caracterização do biossorvente}

A análise da área superficial e porosidade do biossorvente foi determinada pelo método de BET (Brunauer, Emmett \& Teller, 1938) e o diâmetro médio de poros pela técnica de BJH (Barret, Joyner \& Halenda, 1951), em aparelho volumétrico utilizando nitrogênio. A adsorção por nitrogênio, foi sobre uma pressão relativa variando de $10^{-6} \mathrm{a} 1$, por meio do equipamento Quantachrome autosorb-1-CMS-1. Antes da análise, a amostra foi liberada com fluxo de nitrogênio a $105^{\circ} \mathrm{C}$ por $20 \mathrm{~h}$.

\subsection{Ensaios de biossorção em batelada}

Nos ensaios de adsorção foram avaliados o efeito do $\mathrm{pH}\left(1\right.$ a 8), temperatura $\left(30,40\right.$ e $\left.50^{\circ} \mathrm{C}\right)$ e velocidade de agitação (30, 60 e 90 rpm). Os testes foram realizados com 0,3 g de bagaço de mandioca em contato com $50 \mathrm{~mL}$ da solução do corante vermelho escarlate direto à $100 \mathrm{mg} \mathrm{L}^{-1}$. $\mathrm{O} \mathrm{pH}$ foi ajustado com soluções de $0,1 \mathrm{~mol} \mathrm{~L}^{-1} \mathrm{HCl}$ ou $\mathrm{NaOH}$. A mistura foi mantida em agitação em um incubador shaker (SPLabor, SP-222, Brazil) por 5 h. Após, as amostras foram centrifugadas (Quimis, Q222T, Brazil), e encaminhadas para a leitura em espectrofotômetro (Varian, Cary 50 Scan, United States) em $495 \mathrm{~nm}$. A quantidade de corante adsorvida foi determinada de acordo com a Equação 1.

$$
q=\frac{V\left(c_{0}-c\right)}{m}
$$

$\mathrm{C}_{0}$ e $\mathrm{C}\left(\mathrm{mg} \mathrm{L}^{-1}\right)$ : concentração inicial e final do corante na solução aquosa, respectivamente; $\mathrm{m}$ (g): massa de biossorvente; V

$$
\text { (L): volume de solução do corante. }
$$

Os ensaios de equilíbrio e cinético foram realizados em triplicata no sistema batelada, nas melhores condições obtidas nos testes preliminares. A cinética utilizou $0,3 \mathrm{~g}$ de adsorvente e $50 \mathrm{~mL}$ de solução de $100 \mathrm{mg} \mathrm{L}^{-1}$ de corante vermelho escarlate direto, nas condições operacionais de $\mathrm{pH} 2,50^{\circ} \mathrm{C}$ e $90 \mathrm{rpm}$, sendo alíquotas retiradas a cada $15 \mathrm{~min}$. Os modelos aplicados para representar a cinética estão representados na Tabela 1, sendo estes os modelos de pseudo-primeira ordem (Lagergren, 1898), pseudo-segunda ordem (Ho \& McKay, 1998) e Elovich (Elovich \& Larinov, 1962), dados pelas Equações 2 a 4, respectivamente.

Tabela 1. Modelos cinéticos utilizados na modelagem matemática dos dados experimentais de adsorção do corante vermelho escarlate direto por bagaço de mandioca.

\begin{tabular}{ccc}
\hline Modelo & Equação \\
\hline Pseudo-primeira ordem & $q(t)=q_{1}\left(1-e^{-k_{1} t}\right)$ \\
\hline Pseudo-segunda ordem & $q(t)=\frac{t}{\left(1 / k_{2} q_{2}{ }^{2}\right)+\left(t / q_{2}\right)}$ \\
\hline Elovich & $q(t)=\frac{1}{\beta} \ln (1+\alpha \beta t)$ \\
\hline
\end{tabular}

Onde: $\mathrm{k}_{1}\left(\mathrm{~min}^{-1}\right)$ e $\mathrm{k}_{2}\left(\mathrm{~g} \mathrm{mg}^{-1} \mathrm{~min}^{-1}\right)$ são as constantes de taxa dos modelos de pseudo-primeira e pseudo-segunda ordem, respectivamente, $\mathrm{q}_{1}$ e q $\left(\mathrm{mg} \mathrm{g}^{-1}\right)$ são os valores teóricos de biossorção da capacidade calculada pelos modelos de de pseudo-primeira e pseudo-segunda ordem, respectivamente, $\alpha\left(\mathrm{mg} \mathrm{g}^{-1} \mathrm{~min}^{-1}\right)$ é a taxa inicial de biossorção e $\beta\left(\mathrm{g} \mathrm{mg}^{-1}\right)$ é a constante de dessorção do modelo de Elovich. Fonte: Autores. 
As curvas de equilíbrio foram construídas com $50 \mathrm{~mL}$ de solução de corante vermelho escarlate direto à $120 \mathrm{mg} \mathrm{L}^{-1}$, variando a massa de biossorvente de 0,02 a 0,60 g, nas condições de $\mathrm{pH} 2,50{ }^{\circ} \mathrm{C}$ e $90 \mathrm{rpm}$. Os parâmetros de equilíbrio foram avaliados de acordo com os modelos de Langmuir (1918), Freundlich (1906), Redlich-Peterson (1959) e Toth (1971), presentes na Tabela 2, de acordo com as Equações 5 e 8, respectivamente.

Tabela 2. Modelos de isotermas de equilíbrio utilizados na modelagem matemática dos dados experimentais de adsorção do corante vermelho escarlate direto por bagaço de mandioca.

\begin{tabular}{cc}
\hline Modelo & Equação \\
\hline Langmuir & $q_{e}=\frac{q_{m} K_{L} C_{e q}}{1+K_{L} C_{e q}}$ \\
\hline Freundlich & $q_{e}=K_{F} C_{e}^{1 / n F}$ \\
\hline Redlich-Peterson & $q_{e}=\frac{K_{R P} C_{e}}{1+\alpha C_{e}^{\beta}}$ \\
\hline Toth & $q_{e}=\frac{q_{m} b_{t o t h} C_{e}}{\left(1+b_{\text {toth }} C_{e}^{n_{T}}\right)^{\frac{1}{n_{T}}}}$ \\
\hline
\end{tabular}

Onde: $\mathrm{q}_{\mathrm{m}}\left(\mathrm{mg} \mathrm{g}^{-1}\right)$ capacidade máxima de biossorção em monocamada, $\mathrm{K}_{\mathrm{L}}\left(\mathrm{L} \mathrm{mg}^{-1}\right), \mathrm{K}_{\mathrm{F}}\left(\mathrm{mg} \mathrm{g}^{-1}\right)\left(\mathrm{mg} \mathrm{L}^{-1}\right)^{-1 / n}, \mathrm{~K}_{\mathrm{RP}}\left(\mathrm{L} \mathrm{g}^{-1}\right), \alpha\left(\mathrm{mg} \mathrm{L}^{-1}\right)^{-\beta}$ e b toth $\left(\mathrm{L} \mathrm{mg}^{-1}\right)$ são as constantes dos modelos e $\mathrm{n}_{\mathrm{F}}, \beta, \mathrm{m}$ e $\mathrm{n}_{\mathrm{T}}$ (adimensioanis) são os expoentes dos modelos, relacionados com a heterogeneidade de adsorção. Fonte: Autores.

A modelagem matemática foi realizada por meio do software Origin 8.0 (Originlab Corporation, EUA).

\section{Resultados e Discussão}

\subsection{Caracterização do bagaço de mandioca}

A Tabela 3, demonstra as isotermas de adsorção/dessorção de nitrogênio para caracterização do bagaço de mandioca.

Tabela 3. Isotermas de adsorção/dessorção de nitrogênio pelos métodos de BET e BJH do bagaço de mandioca.

\begin{tabular}{cc}
\hline Análise & Valores \\
\hline Área superficial específica por BET $\left(\mathrm{m}^{2} \mathrm{~g}^{-1}\right)$ & 3,012 \\
\hline Volume total de poros $\left(\mathrm{cm}^{3} \mathrm{~g}^{-1}\right)$ & $2,184 \times 10^{-3}$ \\
\hline Diâmetro médio de poros $(\AA)$ & 13,553 \\
\hline
\end{tabular}

Fonte: Autores (2021).

Por meio da Tabela 3, observa-se que o diâmetro médio de poros apresentou um valor de 13,553 Å (1,355 nm), indicando que o bagaço de mandioca possui em sua maioria a presença de microporos, segundo a classificação da IUPAC (1991), a qual considera microporos, materiais com diâmetro inferior a $2 \mathrm{~nm}$. A área superficial específica por BET de resíduos agroindustriais em sua forma bruta aplicados na biossorção, apresentaram valores próximo a este trabalho, como nos estudos de Kosasih et al. (2010) e Giraldo et al. (2020), que obtiveram valores de área superficial específica de 3,72 e 5,21 m² $\mathrm{g}^{-1}$, utilizado como biossorvente cascas de mandioca e bagaço de cana de açúcar para remover cobre e mercúrio, respectivamente. 


\subsection{Ensaios de biossorção do corante vermelho escarlate direto por bagaço de mandioca}

As melhores condições obtidas de $\mathrm{pH}$, temperatura e velocidade de rotação dos testes preliminares de biossorção do corante vermelho escarlate direto por bagaço de mandioca, encontram-se apresentadas na Tabela 4.

$\mathrm{O}$ pH da solução, interfere no grau de ionização da molécula do adsorvente e sua carga elétrica superficial (Sun, Sun \& Wang, 2019; Yagub et al., 2014). A condição de pH que proporcionou a maior remoção foi a pH 2, ou seja, em meio ácido. De acordo com Rigueto et al. (2020b), alguns biossorventes podem apresentar inibição do processo adsortivo conforme o aumento do pH, devido a solubilização dos grupos orgânicos presentes na superfície do biossorvente.

Tabela 4. Condições ótimas de operação obtidas nos testes preliminares da adsorção do corante vermelho escarlate direto por bagaço de mandioca.

\begin{tabular}{cccc}
\hline & pH & Temperatura & Velocidade de agitação \\
\hline Condição ótima de operação & 2 & $50{ }^{\circ} \mathrm{C}$ & $90 \mathrm{rpm}$ \\
\hline
\end{tabular}

Fonte: Autores (2021).

A temperatura é um dos parâmetros que influencia no equilíbrio de adsorção, em razão da interferência na agitação da molécula, que afeta as forças eletrostáticas entre o corante e o biossorvente (Marin et al., 2015). A Tabela 4, demostra que a melhor condição de temperatura foi a $50{ }^{\circ} \mathrm{C}$, ou seja, na maior temperatura estudada. Rigueto et al. (2019), obteve o mesmo resultado utilizando cascas de soja tratadas quimicamente com $\mathrm{NaOH}$ na remoção do corante azul reativo BF-5G.

A velocidade de agitação com maior percentual de remoção, apresentada na Tabela 4 foi à 90 rpm, por ser a maior rotação, há um aumento na taxa de adsorção, em função da menor resistência ao fenômeno de transferência de massa por convecção do soluto presente na solução (Honorio et al., 2015). Efeito similar foi relatado por Freitas et al. (2019), o qual verificou aumento da remoção do corante azul reativo BF-5G com o aumento da velocidade de rotação, com melhor condição a $100 \mathrm{rpm}$, utilizado bagaço de laranja como biossorvente. Assim, as melhores condições para a biossorção do corante vermelho escarlate direto por bagaço de mandioca, foram $\mathrm{pH} 2,50{ }^{\circ} \mathrm{C}$ e $90 \mathrm{rpm}$.

As curvas de cinéticas e de equilíbrio encontram-se apresentadas na Figura 1 (a) e (b), respectivamente, para avaliar o efeito do tempo e a capacidade de biossorção do bagaço de mandioca com o corante vermelho escarlate direto.

A curva cinética presente na Figura 1 (a), apresenta uma grande redução da concentração do corante nos primeiros minutos, com tempo de equilíbrio alcançado em 300 min, com remoção do corante de 84\%. No tempo de 300 min, observa-se que a densidade de adsorção continua aumentando, indicando que o biossorvente não está completamente saturado pelo corante adsorvido, situação análoga foi relatada por Rigueto et al. (2021b) na remoção do corante amarelo tartrazina por esferas de gelatina recuperada de resíduos de couro curtido ao cromo (III). 
Figura 1. Biossorção do corante vermelho escarlate direto por bagaço de mandioca $\left(50{ }^{\circ} \mathrm{C}, \mathrm{pH} 2\right.$ e $\left.90 \mathrm{rpm}\right)$. a) Curva cinética e b) Isotermas de equilíbrio.

a)

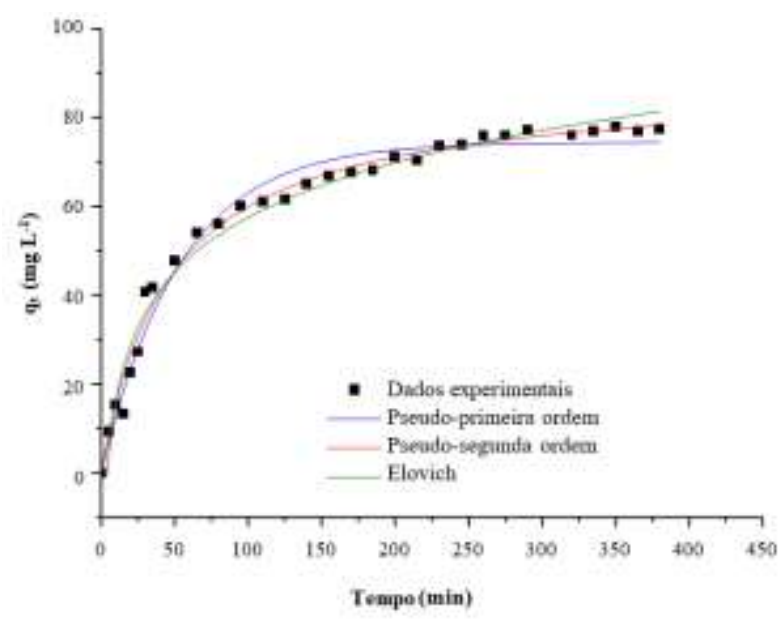

b)

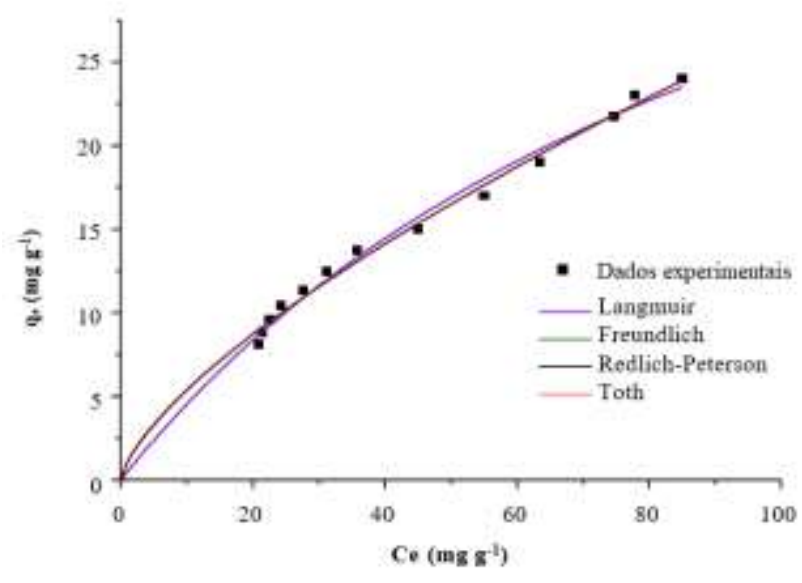

Fonte: Autores (2021).

A curva de equilíbrio mostrada na Figura 1 (b), é classificada de acordo Giles et al. (1960), com perfil L1. A classe L, na parte inicial da curva mostra que há um aumento da concentração do corante que provoca um aumento na capacidade de adorção até a saturação dos sítios ativos, no qual começa ocorrer competividade entra as móleculas de soluto para ocupar os sítos disponíveis, além disso, indica que adsorção ocorre na superfície e por forças fracas, como van der Waals. A subclasse 1 do tipo L, indica que os sítíos ativos não estavam totalmente preenchidos, e o perfil de isoterma L1 geralmente é descrito pelo modelo de Freundlich (Giles et al., 1960; Piccin et al., 2017).

O possível mecanismo que ocorre a adsorção do corante pelo bagaço de mandioca é por meio de interações eletrostáticas, no qual os grupos presentes na superfície da biomassa, são protonados em meio ácido, adquirindo carga superficial positiva, o que provoca atração das moléculas do corante aniônico, que apresenta carga negativa, favorecendo a adsorção (Módenes et al., 2015).

\subsection{Modelagem matemática dos dados experimentais cinéticos e de equilíbrio}

Os parâmetros dos modelos de pseudo-primeira ordem, pseudo-segunda ordem e Elovich ajustados aos dados experimentais, estão apresentados na Tabela 5. Os coeficientes de determinação $\left(\mathrm{R}^{2}\right)$ para ambos os modelos foram superiores a 0,98, demostrando que a adsorção pode obedecer a mais de um mecanismo. O modelo de pseudo primeira-ordem, sugere que a adsorção ocorre por uma força motriz devido a diferença de concentração no coeficiente externo de transferência de massa (Piccin et al., 2012). O bom ajuste dos modelos de Elovich e pseudo segunda-ordem sugerem que a adsorção é por meio de transferência de massa interna e externa, sendo a quimissorção importante, envolvendo troca de elétrons entre o biossorvente e o corante (Piccin et al., 2011; Vargas et al., 2011). 
Tabela 5. Parâmetros dos modelos cinéticos para a biossorção do corante vermelho escarlate direto $\left(50{ }^{\circ} \mathrm{C}, \mathrm{pH} 2\right.$ e $\left.90 \mathrm{rpm}\right)$.

\begin{tabular}{|c|c|c|}
\hline Modelos & Parâmetros & Valores \\
\hline \multirow{3}{*}{ Pseudo-primeira ordem } & $q_{1}\left(\mathrm{mg} \mathrm{g}^{-1}\right)$ & 74,43 \\
\hline & $k_{l}\left(\min ^{-1}\right)$ & 0,018 \\
\hline & $R^{2}$ & 0,98 \\
\hline \multirow{3}{*}{ Pseudo-segunda ordem } & $q_{2}\left(\mathrm{mg} \mathrm{g}^{-1}\right)$ & 77,64 \\
\hline & $k_{2}\left(\mathrm{~g} \mathrm{mg}^{-1} \mathrm{~min}^{-1}\right)$ & 2,46 \\
\hline & $R^{2}$ & 0,99 \\
\hline \multirow{3}{*}{ Elovich } & $\alpha\left(\mathrm{mg} \mathrm{g}^{-1} \min ^{-1}\right)$ & 4,48 \\
\hline & $\beta\left(\mathrm{g} \mathrm{mg}^{-1}\right)$ & 0,056 \\
\hline & $R^{2}$ & 0,98 \\
\hline
\end{tabular}

Fonte: Autores (2021).

A Tabela 6 apresenta os ajustes dos modelos isotérmicos de Langmuir, Freundlich, Redlich-Peterson e Toth aos dados experimentais. Os coeficientes de determinação $\left(\mathrm{R}^{2}\right)$ para ambos os modelos foram superiores a 0,99.

O modelo de Redlich-Peterson é um modelo de três parâmetros que utiliza elementos das equações de Langmuir e Freundlich, quando o parâmetro $\beta$ é igual a 1, a equação é reduzida ao modelo de Langmuir, porém quando este tender a 0 , o modelo se reduz a Freundlich (Foo \& Hameed, 2010). O modelo de Toth é uma equação empírica baseada em melhorar o ajuste da isoterma de Langmuir e descrever sistemas heterogêneos de adsorção, apresentando q $\mathrm{q}_{\mathrm{m}}$ de $24,91 \mathrm{mg}^{-1}$, próximo ao obtido pelo modelo de Langmuir que foi de 25,10 $\mathrm{mg} \mathrm{g}^{-1}$ (Vargas et al., 2011). O parâmetro $\mathrm{n}_{\mathrm{T}}$ do modelo de Toth, prediz a heterogeneidade da adsorção, logo quando igual a 1 se reduz a isoterma de Langmuir e caso contrário, ou seja, se desviar de 1 , tende a um sistema heterogêneo (Ayawei, Ebelegi, \& Wankasi, 2017).

Nesse contexto, analisando os parâmetros dos modelos de Redlich-Peterson e Toth, percebe-se que o valor de $\beta(0,3)$ tende a 0 e o parâmetro $\mathrm{n}_{\mathrm{T}}(0,06)$ se desvia muito de 1 , sugerindo em ambas as análises que o modelo que melhor representa os dados experimentais é o de Freundlich. O modelo de Freundlich sugere que adsorção ocorre em uma superfície heterogênea, com afinidade entre os sítios de adsorção. O parâmetro $\mathrm{n}_{\mathrm{F}}$ representa o fator de heterogeneidade, que se maior que 1 (1,43), indica um processo físico favorável, além disso outra análise pode ser feita por meio da relação $1 / \mathrm{n}_{\mathrm{F}}$, que se menor que 1 , indica uma adsorção normal (Rigueto et al., 2021a; Vargas et al., 2011). 
Tabela 6. Parâmetros dos modelos de equilíbrio para a biossorção do corante vermelho escarlate direto $\left(50{ }^{\circ} \mathrm{C}\right.$, pH 2 e 90 rpm).

\begin{tabular}{|c|c|c|}
\hline Modelos & Parâmetros & Valores \\
\hline \multirow{3}{*}{ Langmuir } & $q_{m}\left(\mathrm{mg} \mathrm{g}^{-1}\right)$ & 25,10 \\
\hline & $K_{L}\left(\mathrm{~L} \mathrm{mg}^{-1}\right)$ & 0,01 \\
\hline & $R^{2}$ & 0,99 \\
\hline \multirow{3}{*}{ Freundlich } & $K_{F}\left(\mathrm{mg} \mathrm{g}^{-1}\right)\left(\mathrm{mg} \mathrm{L}^{-1}\right)^{-1 / \mathrm{n}}$ & 1,08 \\
\hline & $n_{F}$ & 1,43 \\
\hline & $R^{2}$ & 0,99 \\
\hline \multirow{4}{*}{ Redlich-Peterson } & $K_{R P}\left(\mathrm{~L} \mathrm{~g}^{-1}\right)$ & 3,18 \\
\hline & $\alpha\left(\mathrm{L} \mathrm{mg}^{-1}\right)^{-\beta}$ & 57,74 \\
\hline & $B$ & 0,30 \\
\hline & $R^{2}$ & 0,99 \\
\hline \multirow{4}{*}{ Toth } & $q_{m}\left(\mathrm{mg} \mathrm{g}^{-1}\right)$ & 24,91 \\
\hline & $b_{\text {Toth }}\left(\mathrm{L} \mathrm{mg}^{-1}\right)$ & 0,34 \\
\hline & $n_{T}$ & 0,06 \\
\hline & $R^{2}$ & 0,99 \\
\hline
\end{tabular}

Fonte: Autores (2021).

A capacidade máxima de biossorção na monocamada pelo modelo de Langmuir, no presente trabalho foi de $25,1 \mathrm{mg} \mathrm{g}-$ ${ }^{1}$ para a adsorção do corante vermelho escarlate direto por bagaço de mandioca. Outros estudos avaliaram a capacidade de biossorção de resíduos agroindustriais, como Munagapati et al. (2018) e Costa \& Paranhos (2019), na remoção dos corantes preto reativo 5 e vermelho de remazol de solução aquosa utilizando pó de casca de banana e cinzas de casca de arroz, os quais obtiveram valores de $\mathrm{q}_{\mathrm{m}}$ de 49,2 e 11,83 $\mathrm{mg} \mathrm{g}^{-1}$, respectivamente. Por fim, o bagaço de mandioca mostrou-se eficiente na remoção do corante vermelho escarlate direto, tornando-se uma alternativa promissora para remoção de corantes de meio aquoso.

\section{Considerações Finais}

O presente trabalho utilizou bagaço de mandioca como biossorvente para a adsorção do corante têxtil vermelho escarlate direto de solução aquosa. O biossorvente apresentou área superficial específica de $3,012 \mathrm{~m}^{2} \mathrm{~g}^{-1}$ e presença de microporos.

Nos testes preliminares de adsorção as condições ideiais obtidas foram em pH 2, $50{ }^{\circ} \mathrm{C} \mathrm{e} 90 \mathrm{rpm}$. Na cinética, no tempo de 300 min obteve-se uma remoção de 84\%, e nas isotermas de equilíbrio, a capacidade máxima de adsorção em monocamada estimada pelo modelo de Langmuir foi de $25,1 \mathrm{mg} \mathrm{g}^{-1}$.

$\mathrm{Na}$ modelagem matemática, ambos os modelos de Pseudo-primeira ordem, Pseudo-segunda ordem e Elovich representam os dados cinéticos, sugerindo ocorrência de mais de um mecanismo no processo, enquanto que nas isotermas, os modelos de Redlich-Peterson e Toth sugerem uma tendência ao modelo de Freundlich. 
De forma geral, o bagaço de mandioca mostrou-se um adsorvente eficiente na remoção do corante em estudo, porém, ressalta-se a necessidade de novos estudos utilizando o bagaço de mandioca como biossorvente, empregando pré-tratementos e utilizando outros contaminantes para remoção de matrizes aquosas, almejando capacidades de adsorção mais elevadas.

\section{Agradecimentos}

Os autores agradecem à Fundação de Amparo à Pesquisa do Estado de Mato Grosso (FAPEMAT) pelo apoio financeiro.

\section{Referências}

Ahmad, T., \& Danish, M. (2018). Prospects of banana waste utilization in wastewater treatment: A review. Journal of Environmental Management, 206, 330348 .

Alvarado, N., Abarca, R. L., Urdaneta, J., Romero, J., Galotto, M. J., \& Guarda, A. (2021). Cassava starch: structural modification for development of a bioadsorber for aqueous pollutants. Characterization and adsorption studies on methylene blue. Polymer Bulletin, 78, $1087-1107$.

Ayawei, N., Ebelegi, A. N., \& Wankasi, D. (2017). Modelling and interpretation of adsorption isotherms. Journal of Chemistry, 3039817.

Barrett, E. P., Joyner, L. G., \& Halenda, P. P. (1951). The determination of pore volume and area distributions in porous substances. I. computations from nitrogen isotherms. Journal of the American Chemical Society, 73 (1), 373-380.

Brazilian Association of Textile and Clothing Industry. (2019). Sector Profile. https://www.abit.org.br/cont/perfil-do-setor.

Brunauer, S., Emmett, P. H., \& Teller, E. (1938). Adsorption of gases in multimolecular layers. Journal of the American Chemical Society, 60 (2), 309-319.

Costa, J. A. S., \& Paranhos, C. M. (2019). Evaluation of rice husk ash in adsorption of Remazol Red dye from aqueous media. SN Applied Sciences, 1, 397

Dotto, G. L., \& McKay, G. (2020). Current scenario and challenges in adsorption for water treatment. Journal of Environmental Chemical Engineering, 8 (4), 103988.

Elovich, S. Y., \& Larinov, O. G. (1962). Theory of adsorption from solutions of non electrolytes on solid (I) equation adsorption from solutions and the analysis of its simplest form, (II) verification of the equation of adsorption isotherm from solutions. Izvestiya Akademii Nauk. SSSR, Otdelenie Khimicheskikh Nauk, 2, 209-216.

Escaramboni, B., Núñez, E. G. F., Carvalho, A. F. A., \& Neto, P. O. (2018). Ethanol biosynthesis by fast hydrolysis of cassava bagasse using fungal amylases produced in optimized conditions. Industrial Crops and Products, 112, 368-377.

FAO (Food and Agriculture Organization of the United Nations). (2019). Production quantity of cassava in Brazil. http://www.fao.org/faostat/en/\#data/QC.

Foo, K. Y., \& Hameed, B. H. (2010). Insights into the modeling of adsorption isotherm systems. Chemical Engineering Journal, 156, 2-10.

Freitas, T. S. M., Rigueto, C. V. T., Geraldi, C. A. Q., Loss, R. A., Guedes, S. F., Aranda, D. A. G., Muchave, G. J., \& Gonçalves, J. A. (2019). Biosorption of orange bagasse (Citrus sinensis L. Osbeck) in the removal of reactive blue 5G dye. Engevista, 21 (2), 256-266.

Freundlich, H. M. F. (1906). Over the Adsorption in Solution. The Journal of Physical Chemistry, 57, 385-471.

Giles, C. H., MacEwan, T. H., Nakhwa, S. N., \& Smith, D. (1960). A system of classification of solution adsorption isotherms, and its use in diagnosis of adsorption mechanisms and in measurement of specific surface areas of solids. Journal of the Chemical Society, 111, 3973-3993.

Giraldo, S., Robles, I., Ramirez, A., Flórez, E., \& Acelas, N. (2020). Mercury removal from wastewater using agroindustrial waste adsorbents. SN Applied Sciences, 2, 1029.

Gita, S., Hussan, A., \& Choudhury, T.G. (2017). Impact of textile dyes waste on aquatic environments and its treatment. Environment Ecology, 35, $2349-2353$.

Ho, Y. S., \& Mckay, G. (1998). Kinetic models for the sorption of dye from aqueous solution by wood. Process Safety and Environmental Protection, 76 (2), 183-191.

Honorio, J. F., Veit, M. T., Gonçalvez, G. C., Campos, E. A., \& Fagundes-Klen, M. R. (2016). Adsorption of reactive blue BF-5G dye by soybean hulls: kinetics, equilibrium and influencing factors. Water Science \& Technology, 73 (5), 1166-1174.

IUPAC. (1991). Manual on Catalyst Characterization. Pure and Applied Chemistry, 63, 1227-1246.

Kosasih, A. N., Febrianto, J., Sunarso, J., Ju, Y., Indraswati, \& Ismadji, S. (2010). Sequestering of Cu(II) from aqueous solution using cassava peel (Manihot esculenta). Journal of Hazardous Materials, 180 (1-3), 366-374.

Lagergren, S. (1898). About the theory of so-called adsorption of soluble substances. Kungliga Svenska VetenskapsAkademiens Handlingar, 24 (4), 1-39.

Langmuir, I. (1918). The adsorption of gases on plane surfaces of glass, mica and platinum. Journal of the American Chemical Society, 40, $1361-1403$. 
Marin, P., Borba, C. E., Módenes, A. N., Oliveira, S. P. D., Figueiredo, L. S., \& Passaia, N. (2015). Avaliação do efeito da temperatura, pH e granulometria do adsorvente na adsorção do corante azul reativo $5 \mathrm{~g}$. Engevista, 17, 59-68.

Marques, B. S., Frantz, T. S., Cadaval Junior, T. R. S., Pinto, L. A. A., \& Dotto, G. L. (2019). Adsorption of a textile dye onto piaçava fibers: kinetic, equilibrium, thermodynamics, and application in simulated effluents. Environmental Science and Pollution Research, 26, 28584-28592.

Módenes, A. N., Espinoza-Quiñones, F. R., Geraldi, C. A. Q., Manenti, D. R., Trigueros, D. E. G., Oliveira, A. P., Borba, C. E., \& Kroumov, A. D. (2015). Assessment of the banana pseudostem as a low-cost biosorbent for the removal of reactive blue 5G dye. Environmental Technology, 36 (22), $2892-2902$.

Moussavi, G., \& Mahmoudi, M. (2009). Removal of azo and anthraquinone reactive dyes from industrial wastewaters using MgO nanoparticles. Journal of Hazardous Materials, 168, 806-812.

Munagapati, V. S., Yarramuthi, V., Kim, Y., Lee, K. M., \& Kim, D. (2018). Removal of anionic dyes (Reactive Black 5 and Congo Red) from aqueous solutions using Banana Peel Powder as an adsorbent. Ecotoxicology and Environmental Safety, 148, 601-607.

Pandey, A., Soccol, C. R., Nigam, P., Soccol, V. T., Vandenberghe, L. P. S., \& Mohan, R. (2000). Biotechnological potential of agro-industrial residues. II: cassava bagasse. Bioresource Technology, 74 (1), 81-87.

Piccin, J. S., Cadaval Jr, T. R. S., Pinto, L. A. A., \& Dotto, G. L. (2017). Adsorption isotherms in liquid phase: experimental, modeling, and interpretations. In: Bonilla-Petriciolet, A., Mendonza-Castillo, \& A. D. I., Reynel-Ávila, H.E. (Orgs.). Adsorption processes for water treatment and purification. Cham: Springer, $19-51$.

Piccin, J. S., Dotto, G. L., Vieira, M. L. G., \& Pinto, L. A. A. (2011). Kinetics and mechanism of the food dye FD\&C red 40 adsorption onto chitosan. Journal of Chemical \& Engineering Data, 56, 3759-3765.

Piccin, J. S., Gomes, C. S., Ferris, L. A., \& Gutterres, M. (2012). Kinetics and isotherms of leather dye adsorption by tannery solid waste. Chemical Engineering Journal, 183, 30-38.

Polachini, T. C., Betiol, L. F. L., Lopes-Filho, J. F., \& Telis-Romero, J. (2016). Water adsorption isotherms and thermodynamic properties of cassava bagasse. Thermochimica Acta, 632, 79-85.

Redlich, O., \& Peterson, D. L. (1959). A useful adsorption isotherm. The Journal of Physical Chemistry, 63 (6), 1024.

Rigueto, C. V. T., Fonseca, F. C. A., Zanella, B. B., Rosseto, M., Piccin, J. S., Dettmer, A., \& Geraldi, C. A. Q. (2019). Adsorption study with NaOH chemically treated soybean hull for textile dye removal. Revista Ibero-Americana de Ciências Ambientais, 10 (5), 161-168.

Rigueto, C. V. T., Nazari, M. T., Rosseto, M., Massuda, L. A., Alessandretti, I., Piccin, J. S., \& Dettmer, A. (2021)b. Emerging contaminants adsorption by beads from chromium (III) tanned leather waste recovered gelatin. Journal of Molecular Liquids, 330, 115638.

Rigueto, C. V. T., Nazari, M. T., Souza, C. F., Cadore, J. S., Brião, V. B., \& Piccin, J. S. (2020)a. Alternative techniques for caffeine removal from wastewater: An overview of opportunities and challenges. Journal of Water Process Engineering, 35, 101231.

Rigueto, C. V. T., Piccin, J. S., Dettmer, A., Rosseto, M., Dotto, G. L., Schmitz, A. P. O., Perondi, D., Freitas, T. S. M., Loss, R. A., \& Geraldi, C. A. Q. (2020)b. Water hyacinth (Eichhornia crassipes) roots, an amazon natural waste, as an alternative biosorbent to uptake a reactive textile dye from aqueous solutions. Ecological Engineering, 150, 105817.

Rigueto, C. V. T., Rosseto, M., Nazari, M. T., Ostwald, B. E. P., Alessandretti, I., Manera, C., Piccin, J. S., \& Dettmer, A. (2021)a. Adsorption of diclofenac sodium by composite beads prepared from tannery wastes-derived gelatin and carbon nanotubes. Journal of Environmental Chemical Engineering, 9(1), 105030.

Sun, W., Sun., W., \& Wang, Y. (2019). Biosorption of Direct Fast Scarlet 4BS from aqueous solution using the green-tide-causing marine algae Enteromorpha prolifera. Spectrochimica Acta Part A: Molecular and Biomolecular Spectroscopy, 223, 117347.

Toth, J. (1971). State equations of the solid gas interface layer. Acta Chimica Academiae Scientiarum Hungaricae, 69, $311-317$.

Vargas, A. M. M., Cazetta, A. L., Kunita, M. H., Silva, T. L., \& Almeida, V. C. (2011). Adsorption of methylene blue on activated carbon produced from flamboyant pods (Delonix regia): Study of adsorption isotherms and kinetic models. Chemical Engineering Journal, 168, 722-730.

Yagub, M. T., Sen, T. K., Afroze, S., \& Ang, H. M. (2014). Dye and its removal from aqueous solution by adsorption: A review. Advances in Colloid and Interface Science, 209, 172-184.

Zhuo, N., Lan, Y., Yang, W., Yang, Z., Li, X., Zhou, X., Liu, Y., Shen, J., \& Zhang, X. (2017) Adsorption of three selected pharmaceuticals and personal care products (PPCPs) onto MIL-101(Cr)/natural polymer composite beads. Separation and Purification Technology, 177, $272-280$. 